\title{
Parametric Stability Assessment of Single-phase Grid-tied VSCs Using Peak and Average dc Voltage Control
}

Zhang, Chen; Isobe, Takanori; Suul, Jon Are; Dragicevic, Tomislav; Molinas, Marta

Published in:

IEEE Transactions on Industrial Electronics

Link to article, DOI:

10.1109/TIE.2021.3068551

Publication date:

2021

Document Version

Peer reviewed version

Link back to DTU Orbit

Citation (APA):

Zhang, C., Isobe, T., Suul, J. A., Dragicevic, T., \& Molinas, M. (2021). Parametric Stability Assessment of Singlephase Grid-tied VSCs Using Peak and Average dc Voltage Control. IEEE Transactions on Industrial Electronics, 69(3), 2904 - 2915. https://doi.org/10.1109/TIE.2021.3068551

\section{General rights}

Copyright and moral rights for the publications made accessible in the public portal are retained by the authors and/or other copyright owners and it is a condition of accessing publications that users recognise and abide by the legal requirements associated with these rights.

- Users may download and print one copy of any publication from the public portal for the purpose of private study or research.

- You may not further distribute the material or use it for any profit-making activity or commercial gain

- You may freely distribute the URL identifying the publication in the public portal 


\title{
Parametric Stability Assessment of Single- phase Grid-tied VSCs Using Peak and Average dc Voltage Control
}

\author{
Chen Zhang, Takanori Isobe, Jon Are Suul, Tomislav Dragičević \\ and Marta Molinas
}

\begin{abstract}
A type of peak-value dc voltage control (denoted as PK control) was proposed in the literature for supporting the use of a smaller dc-side capacitance when the single-phase Voltage Source Converter (VSC) is operated as a Static Synchronous Compensator (STATCOM). Although it was demonstrated to operate stably under several conditions, it will be revealed in this paper how the PK control will suffer from a more severe small-signal stability issue under non-ideal grid conditions than the conventional method of controlling the averagevalue of the dc voltage (denoted as Avr control). Especially, it will be shown how the PK control is sensitive to some of the control parameters. To obtain these results, a parameter-oriented stability analysis method is developed in the linear-time periodic (LTP) framework. Then, it is utilized for parametric stability assessments of the Avr and PK control. Finally, both frequency- and time-domain experimental results verified the effectiveness and accuracy of the applied method in the presented analysis.
\end{abstract}

Index Terms-dc voltage control, LTP, small-signal modeling, stability, STATCOM, VSC

\section{INTRODUCTION}

$\mathrm{R}_{\mathrm{r}}^{\mathrm{t}}$ ECENT experience in operating wind farms [1] and photovoltaic (PV) power plants [2] have shown that Voltage Source Converters (VSCs) are prone to smallsignal instability when connected to weak grids. Numerous works have been conducted in this respect using the impedancebased approach [3]-[5], and a variety of VSC impedance models have been proposed [6]-[11]. Such models have served not only for stability analysis but also for revealing the frequency-domain characteristics of VSCs, e.g., the frequency coupling effects [9], [10], and the properties of $d q$ (a)symmetry [12], [13]. Although these earlier works are useful for understanding various stability issues of VSC, most of them are fulfilled in the linear time-invariant (LTI) framework, where the system's steady-state is assumed to be time-invariant and constant. The premise of a time-invariant representation is hard to achieve for many converter systems, including single-phase VSCs, in which the system's steady-state is generally

This work is supported by Energy Technology Development and Demonstration Program (EUDP) (ACTION project, grant No. 56537).

C. Zhang and T. Dragičević are with the Department of Electrical Engineering, Technical University of Denmark (DTU), Denmark, (email: chezh@elektro.dtu.dk; tomdr@elektro.dtu.dk).

T. Isobe is with the Faculty of Pure and Applied Sciences, University of Tsukuba, Japan (email: isobe.takanori.gf@u.tsukuba.ac.jp). characterized by periodic trajectories, i.e., depending on a periodic steady-state (PSS) system representation.

To cope with the modeling and analysis of PSS systems, the linear time-periodic (LTP) method [14], [15] can be applied. An early but heuristic application of the method in identifying and analyzing the harmonic interaction of converters in electrical railways was presented in [16]. Lately, this method has become appealing for modeling and stability analysis of converters with inherent PSS characteristics, e.g., the modular multilevel converters (MMCs) [17], [18], and the single-phase VSCs [19]. Despite the superior applicability of the LTP method, it is not as easy to apply as the LTI method. Hence, in many cases, the LTI method is still attractive when the time varying effects are not evident or can be neglected under certain conditions. E.g., if the double grid-frequency oscillations in the dc voltage of a single-phase VSC system is small [20], it can be modeled similarly as the three-phase VSC using the LTI method [20][23]. However, this simplification may lead to inaccurate results when the PSS effect is substantial [24], e.g., the singlephase VSC equipped with a small dc-side capacitor, which is the scenario considered for the analysis presented in this work.

This paper is addressing the stability analysis and comparison of two types of dc voltage control strategies applied to a single-phase grid-tied VSC operated as a Static Synchronous Compensator (STATCOM). In this application, the peak-value of the dc-side capacitor voltage will be synchronized with the peak value of the maximum required acside output voltage when the converter is injecting reactive power to the grid [25]. This allows for operating the singlephase STATCOM with reduced dc-side capacitance and correspondingly larger double grid-frequency oscillations of the dc voltage without increasing the peak value of the capacitor voltage. However, an explicit estimation and control of the peak-value of dc voltage is required, rather than the commonly applied approach of controlling the average value of the dc voltage [21] (denoted as "Avr control" for brevity). For this purpose, [26] presented a second-order generalized-integrator (SOGI)-based filter for estimating the peak-value of the dc voltage. The resulting dc voltage control scheme is briefly denoted by "PK control" in later analysis.

J. A. Suul is with SINTEF Energy Research, also with the Department of Engineering Cybernetics, Norwegian University of Science and Technology (NTNU), Norway (e-mail: jon.a.suul@sintef.no).

$\mathrm{M}$. Molinas is with the Department of Engineering Cybernetics, NTNU, Norway (email: marta.molinas@ntnu.no) 


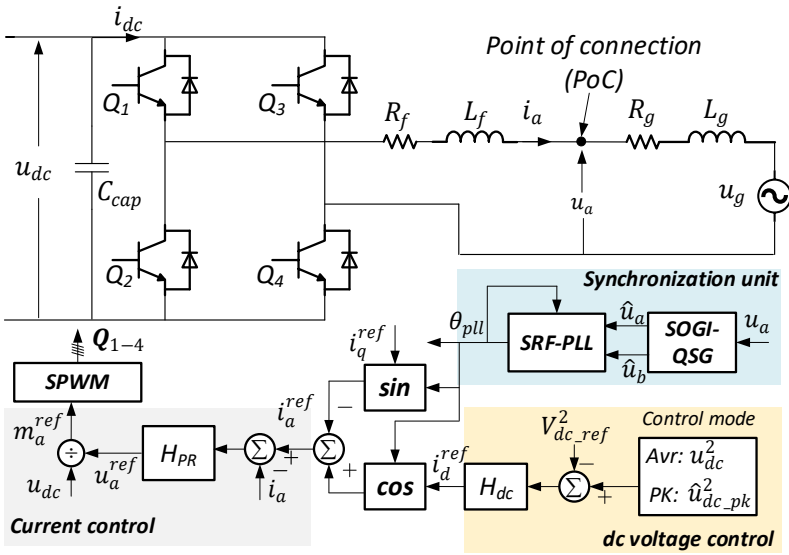

(a)

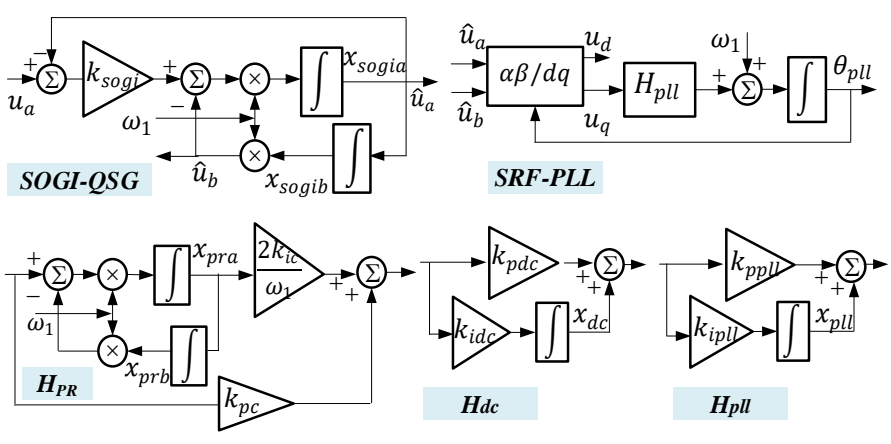

(b)

Fig. 1 The single-phase grid-tied VSC operated as the STATCOM mode. (a) Main circuit and control strategies. (b) Control blocks

Although the PK control introduced in [26] was shown to operate stably with a small dc-side capacitance, the result was obtained under ideal grid conditions. As will be revealed in this paper, the PK control will suffer from a more limited stability range under non-ideal grids than the Avr control. Therefore, aside from the above-mentioned merits of PK control in enabling operation with reduced dc-side capacitance, an indepth evaluation of PK control in view of stability is desired. To this end, this paper will contribute to the following aspects:

1) Developing a parameter-oriented stability assessment method in the LTP framework, which will serve as a tool for fast and efficient parametric stability assessment of this paper.

2) Revealing and discussing the potential stability issues of the PK control provoked by non-ideal grid conditions.

3) Comparing and clarifying the stability performance of the Avr and PK control over a wide parameter space.

\section{Parameter-ORIENTEd Stability ANALYSIS Method APPLIED TO THE SINGLE-PHASE GRID-VSC SYSTEM}

\section{A. Study system}

As shown in Fig. 1 (a), the control system of the single-phase STATCOM mainly consists of: the proportional resonant (PR)based current controller, the proportional integrator (PI)-based dc voltage controller, and the grid-synchronization unit. The synchronization strategy is relying on a SOGI-based Quadrature Signal Generator (QSG) and a Synchronous Reference Frame (SRF) phase-locked-loop (PLL). Detailed information on the control blocks is given in Fig. 1 (b). The aforementioned dc voltage control strategies are listed below:

1) the Avr control, where the square of the measured $\mathrm{dc}$ voltage $u_{\mathrm{dc}}^{2}(t)$ is directly regulated, as shown in Fig. 1 (a);

2) the PK control, where the estimated peak value of the squared dc voltage $\hat{u}_{\mathrm{dc} \_ \text {pk }}^{2}(t)$ (see Fig. 2 (a)) is regulated.

In Fig. 2 (b), time-domain dc voltage waveforms under the Avr and the PK control are given to better illustrate their basic control effects. It can be seen that the Avr control can only effectively regulate the average value of the actual dc voltage, leaving the twice grid-frequency ripple uncontrolled. When the reactive current injection is large, the amplitude of the capacitor voltage oscillations will increase correspondingly, thus a larger dc capacitor has to be equipped to prevent the system from potential overvoltage. By contrast, the PK control can effectively limit the peak-value of dc voltage to the control reference, thus a smaller dc-side capacitance can be utilized.

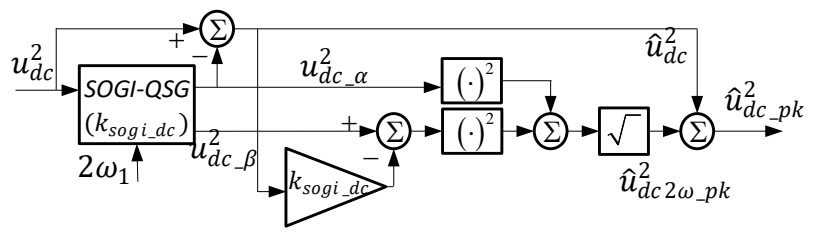

(a) A SOGI-based filter for detecting the peak value of dc voltage [26]

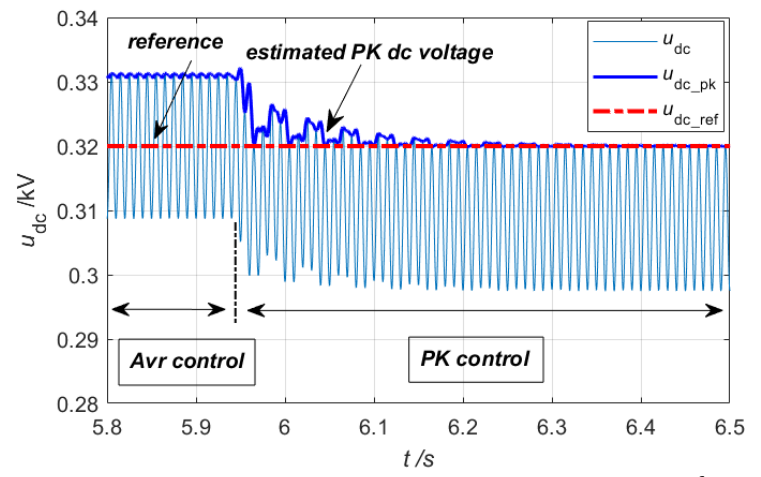

(b) DC voltage waveforms under the Avr and PK controls $\left(i_{q}^{\text {ref }}=-3 A\right.$ )

Fig. 2 Peak dc voltage detection and control effects

It is also worth mentioning that the above-mentioned merit of PK control mainly applies to the STATCOM operation mode, because controlling the peak-value of the dc voltage will typically lead to a lowered average dc voltage at high loads (see Fig. 2 (b)). This trait is not feasible for supplying active power loads. For attenuating the dc voltage ripples in the context of supplying active power loads, one may refer to [27] for details. After clarifying the study system, next, the parameter-oriented stability analysis method will be developed and introduced.

\section{B. Brief introduction of the LTP method}

For a nonlinear system, expressed as

$$
\begin{aligned}
& \dot{\boldsymbol{x}}=\boldsymbol{f}(t, \boldsymbol{x}, \boldsymbol{u}), \\
& \boldsymbol{y}=\boldsymbol{g}(t, \boldsymbol{x}, \boldsymbol{u})
\end{aligned},
$$

linearizing (1) around its PSS conditions $x_{0}(t)$ will typically lead to an LTP system, expressed as 


$$
\begin{aligned}
& \Delta \dot{\boldsymbol{x}}=A(t) \Delta \boldsymbol{x}+B(t) \Delta \boldsymbol{u} \\
& \Delta \boldsymbol{y}=C(t) \Delta \boldsymbol{x}+D(t) \Delta \boldsymbol{u}
\end{aligned}
$$

where $A(t)=\left.\frac{\partial \boldsymbol{f}}{\partial \boldsymbol{x}}\right|_{x_{0}(t)}, B(t)=\left.\frac{\partial \boldsymbol{f}}{\partial \boldsymbol{u}}\right|_{\boldsymbol{u}_{0}(t)}, C(t)=\left.\frac{\partial \boldsymbol{g}}{\partial \boldsymbol{x}}\right|_{x_{0}(t)}$, and $D(t)=\left.\frac{\partial \boldsymbol{g}}{\partial \boldsymbol{u}}\right|_{\boldsymbol{u}_{0}(t)}$, while $\boldsymbol{u}_{0}(t)$ is the system's steady-state input vector. Stability analysis of (2) can be typically performed in two ways: 1) use of the Floquet theory [14], where the eigenvalue of a so-called monodromy matrix obtained from a $T$ period numeric integration of $\boldsymbol{A}(t)$ is analyzed; 2) apply the Hill's frequency-domain method [14], where the eigenvalue of a so-called harmonic-state-space (HSS) model expressed by (3) is evaluated (i.e., the eigenvalues of the matrix $\mathcal{A}-\mathcal{N}_{\text {blk }}$ ) [19].

$$
\begin{aligned}
& \Delta \dot{\mathcal{X}}=\left(\mathcal{A}-\mathcal{N}_{\text {blk }}\right) \Delta \mathcal{X}+\mathcal{B} \Delta \mathcal{U} \\
& \Delta \mathcal{Y}=\mathcal{C} \Delta \mathcal{X}+\mathcal{D} \Delta \mathcal{U}
\end{aligned}
$$

where $\mathcal{N}_{\text {blk }}=\operatorname{diag}\left\{-\mathrm{j} k \omega_{1} \boldsymbol{I}, \ldots, 0, \ldots, \mathrm{j} k \omega_{1} \boldsymbol{I}\right\}$ is a block diagonal matrix; $\omega_{1}$ is the fundamental frequency; $I$ is an identity matrix with the same dimension as the state vector $\boldsymbol{x}$; $k \in[-N, N]$ is defined for numeric implementation, and $N$ is the highest harmonic-order (its value will be discussed later). $\Delta \mathcal{X}, \Delta \mathcal{U}, \Delta \mathcal{Y}$ are spectral vectors collecting the Fourier coefficients of vectors $\Delta \boldsymbol{x}, \Delta \boldsymbol{u}, \Delta \boldsymbol{y} \quad, \quad$ e.g., $\Delta \mathcal{X}=\left[\boldsymbol{X}_{-k}, \ldots, \boldsymbol{X}_{0}, \ldots, \boldsymbol{X}_{+k}\right]^{T}$ and the element $\boldsymbol{X}_{+k}$ denotes the $k$ th Fourier coefficient of $\Delta x$. This definition also applies to $\Delta \mathcal{U}$ and $\Delta \mathcal{Y}$. At last, $\mathcal{A}, \mathcal{B}, \mathcal{C}, \mathcal{D}$ are Toeplitz formatted matrices of $A(t), B(t), C(t), D(t)$.

It should be noted that both methods rely on the knowledge of $\boldsymbol{x}_{0}(t)$ or its Fourier coefficients $\mathcal{X}$. This means that the PSS conditions should be obtained beforehand, which is usually assisted by time-domain simulations, i.e., via numeric integration of the system (1). However, the simulation-based PSS extraction method has two main drawbacks when applied to parametric studies: 1) changing the system's parameters will typically lead to a new set of PSS conditions, which need to be updated by running a new simulation and the process of which is time-consuming; 2) by running the simulation, only the stable PSS conditions can be extracted. This restricts its application in parametric stability analysis when the parameters should be swept over wide ranges which likely result in the presence of unstable PSS conditions. To overcome these issues and achieve a fast and efficient PSS extraction, the frequency-domain iteration-based approach described in [28] could be adopted.

\section{The iteration-based PSS extraction method}

First, from (1), the $i$-th iterative model can be written as:

$$
\dot{\boldsymbol{x}}^{(i)}+\Delta \dot{\boldsymbol{x}}^{(i)}=\boldsymbol{f}\left(t, \boldsymbol{x}^{(i)}, \boldsymbol{u}^{(i)}\right)+\frac{\partial \boldsymbol{f}}{\partial \boldsymbol{x}^{(i)}} \Delta \boldsymbol{x}^{(i)}+\frac{\partial \boldsymbol{f}}{\partial \boldsymbol{u}^{(i)}} \Delta \boldsymbol{u}^{(i)}
$$

where $A^{(i)}(t)=\frac{\partial f}{\partial x^{(i)}}$ and $B^{(i)}(t)=\frac{\partial f}{\partial \boldsymbol{u}^{(i)}}$. For PSS calculation,

(4) represents a closed-loop system, where $\boldsymbol{u}^{(i)}$ is an input vector consisting of the system's time-invariant parameters, e.g., control references, control parameters, and the magnitude and phase of the supplying source voltage. Since $\boldsymbol{u}^{(i)}$ is known and fixed during the iteration, the last term can be omitted.

Transforming (4) into the frequency-domain and applying the principle of harmonic balance, the $i$-th iterative model for the $k$-th harmonic can be obtained as:

$$
\mathcal{N}_{\text {blk }}\left(\boldsymbol{X}_{k}^{(i)}+\Delta \boldsymbol{X}_{k}^{(i)}\right)=\boldsymbol{F}_{k}^{(i)}+\sum_{m} \boldsymbol{A}_{k-m}^{(i)} \Delta \boldsymbol{X}_{m}^{(i)}
$$

where $\boldsymbol{X}_{k}^{(i)}$ denotes the $k$-th Fourier coefficient of $x$ at the $i$-th step, and $\boldsymbol{F}_{k}^{(i)}=\langle\boldsymbol{f}\rangle_{k}^{(i)}$. The operator $\langle\cdot\rangle_{k}$ is to extract the $k$-th Fourier component of a periodically time-varying function.

Finally, applying (5) to each harmonic and collecting the resulting equations will lead to the final frequency-domain iteration model that can be compactly written as:

$$
\left\{\begin{array}{l}
\Delta \mathcal{X}^{(i)}=\left(\mathcal{A}^{(i)}-\mathcal{N}_{\text {blk }}\right)^{-1}\left(\mathcal{N}_{\text {blk }} \mathcal{X}^{(i)}-\mathcal{F}^{(i)}\right) \\
\mathcal{X}^{(i+1)}=\mathcal{X}^{(i)}+\Delta \mathcal{X}^{(i)}
\end{array}\right.
$$

where $\mathcal{F}^{(i)}=\left[\boldsymbol{F}_{-k}, \ldots, \boldsymbol{F}_{0}, \ldots, \boldsymbol{F}_{+k}\right]^{T}$ is the collection of Fourier coefficients of $\boldsymbol{f}\left(t, \boldsymbol{x}^{(i)}, \boldsymbol{u}^{(i)}\right)$. To solve (6) numerically, the upper boundary of $k \in[-N, N]$ should be determined. In principle, the higher the $N$ the better the precision. However, a large $N$ is computationally expensive and usually not necessary. E.g., since this paper applies the principle of switching-average for modeling the VSC system, the modelled system will be free from switching and sideband harmonics, thus a relatively low $N$ can be considered. In this work, $N=4$ is applied and its validity will be verified by experiments later. Once $N$ is determined, generally, (6) can be solved by iterations (e.g., the Newton's method) until a pre-defined tolerance is reached.

Next, this method will be implemented in MATLAB for parametric stability analysis. The resulting algorithm will contain three main parts: 1) the model preparation subroutine; 2 ) the numeric iteration subroutine, and 3 ) the main routine for parameter sweeps. They will be elaborated below.

\section{Algorithmic implementation of the method for parametric stability analysis}

1) The model preparation subroutine

To achieve a tool that is easy and efficient to apply, the system modeling is automatized by using symbolic calculations of MATLAB, where the following steps will take place to obtain all the necessary time-domain functions for study:

First, define and formulate the closed-loop system model (1) using the command syms (). Then, perform a system-wide linearization on (1) using the command jacobian(), so that $A(t), B(t), C(t), D(t)$ are further obtained. Finally, parameterize these functions using the command matlabFunction () for better numeric performance when interfacing them with the next subroutine. It is also worth mentioning that, for this work, this subroutine only needs to be run once as the structure of the system is fixed.

\section{2) The numeric iteration subroutine}

This subroutine aims to solve (6) using Newton's method. However, from the above analysis, it can be seen that solving (6) requires time-frequency domain transformation of variables and functions. For which, numerical evaluation of those time- 
domain functions with a duration of $T_{\text {eval }}$ (i.e., usually set as the system' period) and a time-step $h_{\text {eval }}$ will be executed internally. The detailed steps fulfilling this subroutine include:

Step 1: Numerical evaluation of functions (e.g., $A^{(i)}(t)$ with $T_{\text {eval }}$ and $h_{\text {eval }}$ ) using a previously obtained $\boldsymbol{x}^{(i)}$ (or initial states);

Step 2: Transform the results into frequency-domain and collect $\mathcal{A}^{(i)}, \mathcal{F}^{(i)}, \mathcal{X}^{(i)}$ for updating $\mathcal{X}^{(i)} \rightarrow \mathcal{X}^{(i+1)}$ according to (6). Afterward, apply the inverse Fourier transform to find $\boldsymbol{x}^{(i+1)}$, preparing for the next iteration;

Step 3: If $\left\|\Delta \mathcal{X}^{(i)}\right\| \leq \varepsilon$ is met, exit and output $\mathcal{X}^{(i)}$ (or $\left.x_{0}(t)=x^{(i)}\right)$; otherwise, go to Step 1. It should be noted that the outputs of the process also include useful by-products for LTP analyses, e.g., $\mathcal{A}^{(i)}$ is used for later stability analysis, while $\mathcal{A}^{(i)}, \mathcal{B}^{(i)}, \mathcal{C}^{(i)}, \mathcal{D}^{(i)}$ are relevant matrices for generating the VSC's impedance, which will be shown in Section IV.B.

3) The main routine for parameter sweeps

The "iteration subroutine" will find the outputs for a given parameter set. To enable the parametric stability assessment, it is desired to sweep the parameters for a certain range. This can be achieved by adding an outer loop on top of it, where the varying parameters are regarded as the inputs of the parameterized functions obtained in the "model preparation subroutine". Finally, the overall algorithm is obtained, as depicted in Fig. 3. Besides, the main configuration for the "iteration subroutine" applied in this work is given in Table I.

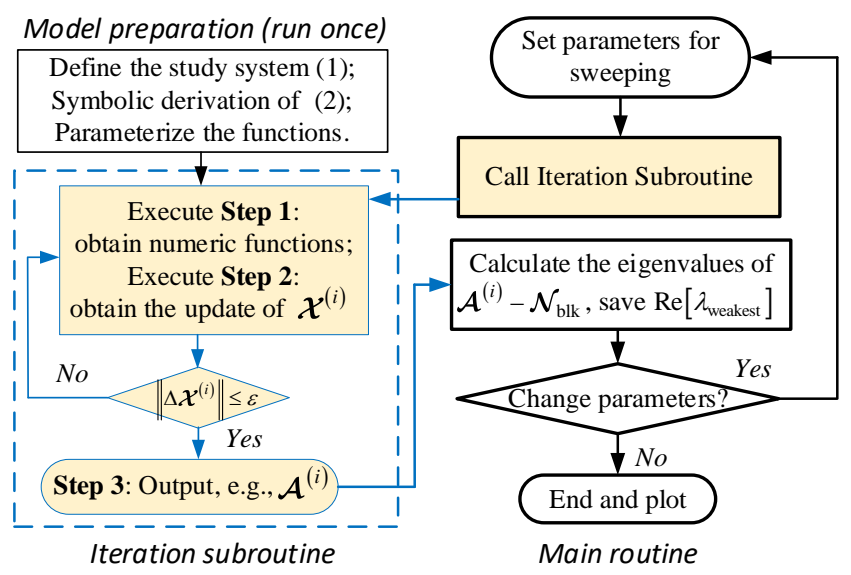

Fig. 3 The flowchart of the parametric stability analysis algorithm

TABLE I MAIN CONFIGURATION FOR ITERATION PROCEDURE

\begin{tabular}{l|l}
\hline \hline Harmonic-order in $N=4$ & Time step: $h_{\text {eval }}=50 \mu \mathrm{s}$ \\
\hline Duration: $T_{\text {eval }}=0.02 \mathrm{~s}$ & Tolerance $\varepsilon=0.001$ \\
\hline $\begin{array}{l}\text { Note: Initial values for iteration are set according to reference values } \\
\text { of controls while the remaining values are filled with zeros }\end{array}$ \\
\hline
\end{tabular}

From the above overall implementation it is noticed that only the closed-loop system model (1) needs to be manually defined in the algorithm for specific applications (for this paper, it corresponds to the closed-loop models of the single-phase STATCOM given in Appendix-A and -B). The remaining calculations (e.g., PSS extraction and linearization) are automatically processed by the algorithm. This shows that the tool could be readily applied to other PSS systems as well.

\section{4) An application example}

Next, application of this algorithm in eigenvalue calculation of the single-phase STATCOM (PK control) for a given parameter set is demonstrated, while the parameter sweepbased analysis will be discussed in next section.

As mentioned, when applying the algorithm, only (1) needs to be manually defined, in this example, it corresponds to (B.4) (see Appendix-B). Then, by running the algorithm, $\mathcal{A}^{(i)}$ can be obtained and the eigenvalues of $\mathcal{A}^{(i)}-\mathcal{N}_{\text {blk }}$ [19] can be further calculated and assessed, the result is shown in Fig. 4. It can be seen that the eigenvalues of an LTP system consist of eigenmodes and their frequency-shifted copies. This is a known trait of LTP systems which seems to complicate the analysis [19]. However, for stability evaluation, the real parts of the eigenmodes closest to the imaginary axis are the most relevant to study. Furthermore, as their real parts are identical, they can be denoted by a unified scalar variable, e.g., $\operatorname{Re}\left[\lambda_{\mathrm{wk}}\right]$ (the subscript denotes the "weakest mode"). In general, the system is stable if $\operatorname{Re}\left[\lambda_{\mathrm{wk}}\right]<0$, otherwise, the system will be unstable. In later analysis, $\operatorname{Re}\left[\lambda_{\mathrm{wk}}\right]$ will serve as the stability index for the parametric stability assessment.

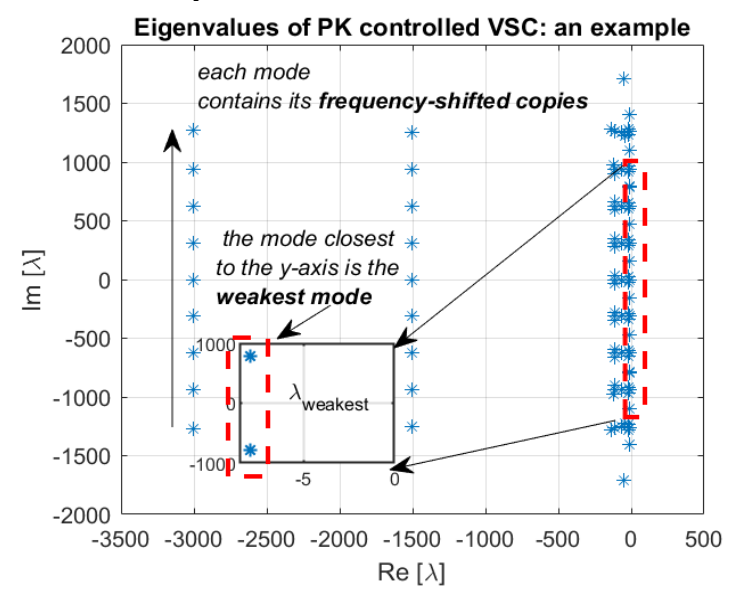

Fig. 4 An application example of eigenvalue calculation

\section{PARAmetric Stability AsSessment Of the Single- PHASE STATCOM UNDER PK AND AVR CONTROLS}

Considering that many VSCs' control parameters are designed under an ideal grid assumption, it is relevant to evaluate how sensitive the stability of the designed system is to changes in grid conditions and which control parameters have the most evident influence on the stability characteristics. To this end, parameter sweeps of Avr and PK controls under ideal and non-ideal grids are conducted, corresponding results will be discussed in Section III.A; while some newly identified issues specific to the PK control will be discussed in Section III.B.

\section{A. Parametric stability analysis of Avr and PK control under ideal and non-ideal grids}

\section{1) Analysis of the Avr control}

According to Fig. 5 (a), it can be seen that the stability characteristics of Avr control under the ideal and non-ideal grids are very close to each other for the parameter sweeps of $i_{q}^{\text {ref }}, \alpha_{p l l}$, and $k_{\text {sogi }}$. As for $\alpha_{c}$, the differences between the 

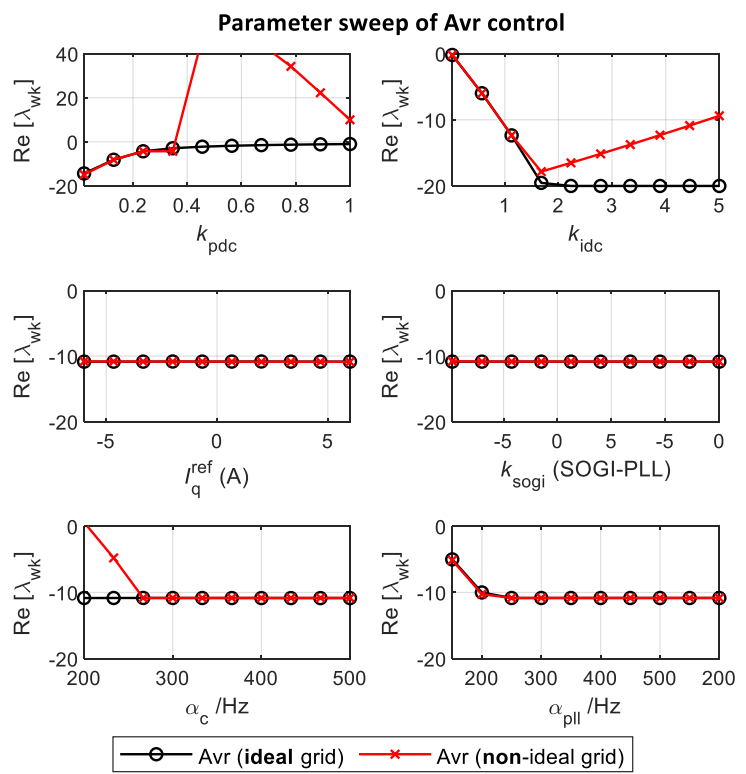

(a)
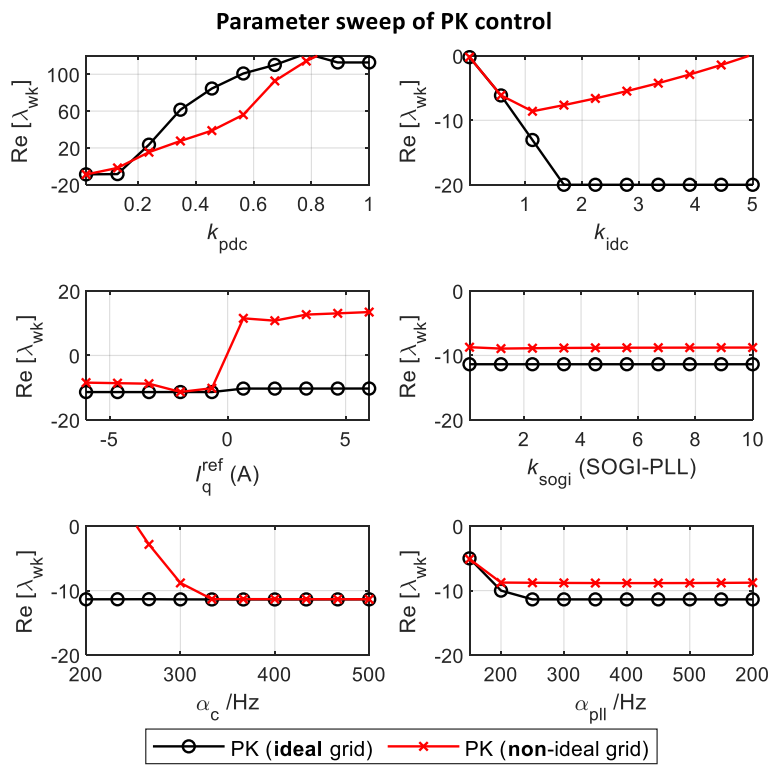

(b)

Fig. 5 Parametric stability analysis of Avr and PK controls under ideal and non-ideal grids. For the ideal grid case, $R_{\mathrm{g}}$ and $L_{\mathrm{g}}$ in Table II are set to zero. When one parameter is swept, other parameters are set to default values given in Table II. Please also note, $k_{\text {sogi_dc }}$ exclusive to the PK control will be discussed later.

ideal and non-ideal grids are negligible when it is greater than a certain value (e.g., $300 \mathrm{~Hz}$, the default value of this paper). Above this value, altering $\alpha_{c}$ will not lead to an evident change of the stability trait (i.e., $\operatorname{Re}\left[\lambda_{\mathrm{wk}}\right]$ ). By contrast, the parameter sweeps of $k_{p d c}$ and $k_{i d c}$ show that not only the stability differences of ideal and non-ideal grids can be large, but also the variations of corresponding stability traits are more evident than those sweeping of $i_{q}^{r e f}, \alpha_{p l l}, k_{\text {sogi }}, \alpha_{c}$ (this is obtained by comparing the variation range of the curves on the $y$-axis).

Table II System parameters and default values

\begin{tabular}{l|l|l|l}
\hline \hline \multicolumn{2}{l|}{ Avr/PK Control parameters } & \multicolumn{2}{c}{ Circuit \& other parameters } \\
\hline Name & Values & Name & Values \\
\hline$k_{p d c} ; k_{i d c}$ & $0.1 ; 1$ & $R_{g} ; L_{g}$ & $0.258 \mathrm{ohm} ; 6.63 \mathrm{mH}$ \\
\hline$i_{q}^{\text {ref }}$ & $-3 \mathrm{~A}$ & $R_{f} ; L_{f}$ & $0.129 \mathrm{ohm} ; 3.3 \mathrm{mH}$ \\
\hline$k_{\text {sogi }}$ & 5 & $C_{c a p}$ & $200 \mu \mathrm{F}$ \\
\hline$\alpha_{c}$ & 300 & $U_{N}$ & $200 \mathrm{~V}(\mathrm{rms})$ \\
\hline$\alpha_{\text {pll }}$ & 20 & $I_{N}$ & $5 \mathrm{~A}(\mathrm{rms})$ \\
\hline$k_{\text {sogi_dc }}(\mathrm{PK})$ & 5 & $V_{d c}^{\text {ref }}$ & $320 \mathrm{~V}$ \\
\hline$\alpha_{c}, \alpha_{p l l}$ in relations with their gains are given in Appendix-C. \\
\hline \hline
\end{tabular}

From the above analysis, it can be further obtained that: 1) the design of $\alpha_{p l l}, k_{\text {sogi }}, \alpha_{c}$ using the ideal grid assumption can be considered "safe" as the resulting stability will not vary too much when applied to a non-ideal grid. Also, the stability is shown less sensitive to their variations compared to $k_{p d c}, k_{i d c}$; 2 ) the design of $k_{p d c}, k_{i d c}$ (in particular for $k_{p d c}$ ) using the ideal grid assumption should be cautious, because the resulting stability characteristics can be very different from the initial design when applied in a non-ideal grid. E.g., as shown in Fig. 5 (a), if $k_{p d c}=0.4$ of the ideal grid design is applied to a nonideal grid, the resulting system will be unstable.

\section{2) Analysis of the PK control}

A similar analysis for the PK control can be obtained from Fig. 5 (b). As seen from the curves, the results for $\alpha_{p l l}, \alpha_{c}$, $k_{\text {sogi }}$, as well as $k_{p d c}$ and $k_{i d c}$ of the Avr control, are all applicable for the case of PK control.

However, in regard to $i_{q}^{r e f}$, an interesting phenomenon emerges under the non-ideal grid, i.e., when the sign of $i_{q}^{r e f}$ is changed (e.g., from injecting to absorbing reactive power), the stability characteristic is drastically changed as well (in fact, deteriorated). It should be noted that this stability issue arising from the reactive current control neither evidently exists in the ideal grid case of the PK control, nor in the Avr control. This can be obtained from their sweeps of $i_{q}^{\text {ref }}$ given in Fig. 5.

\section{3) Stability comparison of Avr and PK controls}

By performing a side-by-side comparison of the values of $\operatorname{Re}\left[\lambda_{\mathrm{wk}}\right]$ between the Avr control and the PK control (qualitatively, the height of the curve on the $y$-axis), it can be obtained that, 1) under the ideal grid, aside from the sweep of $k_{p d c}$, the PK control performs similarly as the Avr control; 2) under the non-ideal grid, the stability margin of the PK control is overall lower than the Avr control (i.e., the curves of PK control are overall higher than those of the Avr control).

This comparative analysis together with the above finding reveals that under non-ideal grids, the single-phase STATCOM operated with the PK control may encounter a more stringent stability limit than the Avr control, even though it can bring benefits in reducing the dc-side capacitance. Therefore, control parameters of the PK control should be carefully designed. This issue will be further discussed by using the stability region.

\section{$B$. Further analysis of the PK control via stability regions}

\section{1) Formulation of the stability region}

Aside from the single parameter sweep analysis, interactions of two parameters and their effects on the small-signal stability characteristics can be analyzed by a three-dimensional plot, e.g., the first plot of Fig. 6. This plot is obtained by first defining a parameter space (i.e., the $x-y$ plane) consisting of two-parameter 
PK: Case I

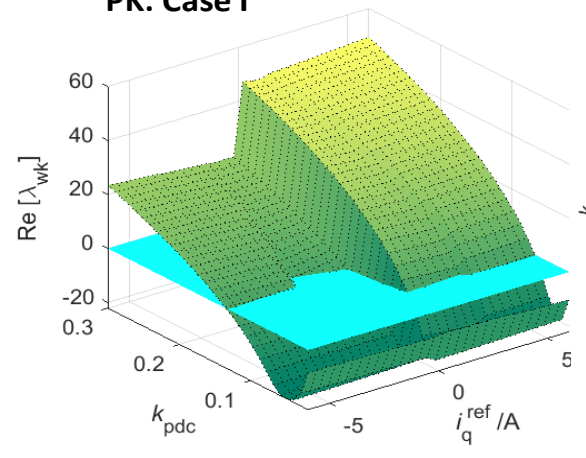

Stability region

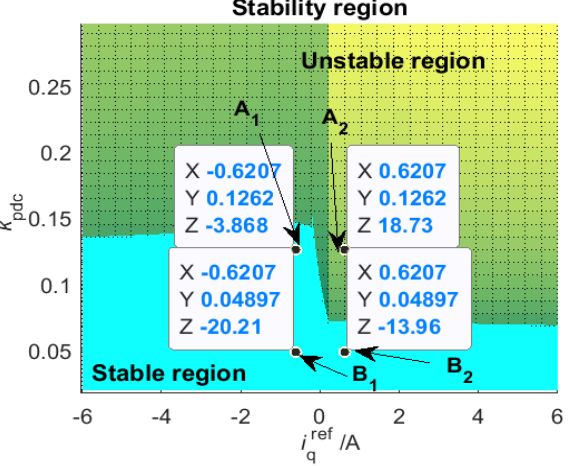

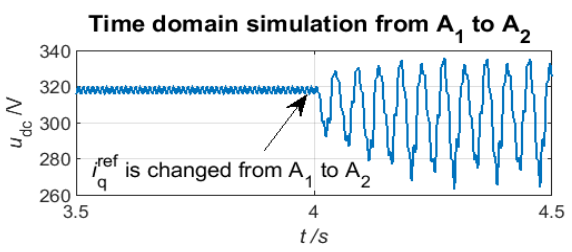

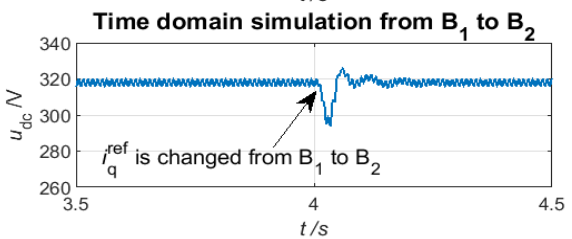

(a) Case I: under parameter space $\left(k_{p d c}, i_{q}^{\text {ref }}\right)$, the rest of parameters are the default values in Table II. The simulation is conducted in PSCAD/EMTDC
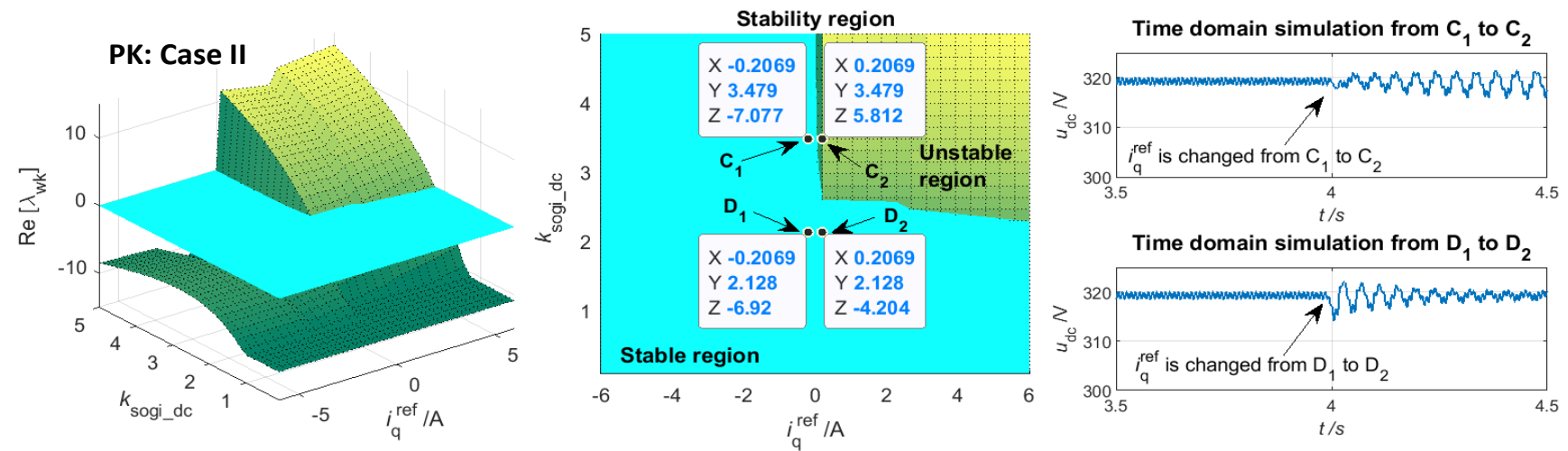

(b) Case II: parameter space $\left(k_{\text {sogi_dc }}, i_{q}^{\text {ref }}\right)$, the rest of parameters are the default values in Table II. The simulation is conducted in PSCAD/EMTDC Fig. 6 Stability region-based analysis and verification of the PK control under two types of parameter space

sweeps, and then, plotting it against the stability index (i.e., the $z$-axis). However, for stability-oriented parameter design and analysis, a parametric map with the indication of stability boundaries would be more useful, see the second plot of Fig. 6 as an example. Such plot is referred to as the stability region plot in this paper, which can be readily obtained by projecting the three-dimensional plot on the plane $z=0$, i.e., projecting the space where $z \leq 0$ will generate the stable region, otherwise, it generates the unstable region.

On the other hand, previous analysis has revealed that $k_{p d c}$ and $i_{q}^{\text {ref }}$ are sensitive parameters for the stability of PK control. Moreover, there is another parameter reserved for discussion, i.e., $k_{\text {sogi_dc }}$. Therefore, this study will focus on these three parameters, for which the following two cases will be analyzed:

1) Case I: under the parameter space $\left(k_{p d c}, i_{q}^{r e f}\right)$;

2) Case II: under the parameter space $\left(k_{\text {sogi_dc }}, i_{q}^{\text {ref }}\right)$.

\section{2) Stability region-based analysis}

First, from the stability region plot of Fig. 6 (a) it can be seen that if $k_{p d c}$ can be kept small, the single-phase STATCOM can stably fulfill a bidirectional reactive current control under the non-ideal grid. Thus, a small $k_{p d c}$ is beneficial for the stability of PK control. This can be justified by simulations given in the last plot of Fig. 6 (a). Indeed, when $i_{q}^{\text {ref }}$ is changed from $\mathrm{A}_{1}$ to $\mathrm{A}_{2}$ under a relatively large $k_{p d c}$, the resulting transition will be unstable. If the same reactive current change occurs under a smaller $k_{p d c}$ (i.e., from $\mathrm{B}_{1}$ to $\mathrm{B}_{2}$ ), the transition becomes stable. These results coincide with the stability region-based analysis.
However, a too-small $k_{p d c}$ may lead to a sluggish response of dc voltage, thus other control parameters can be altered instead to improve the stability. For example, according to the stability region plot of Fig. 6 (b), under the default parameters of the dc voltage controller, the PK control can achieve a stable and bidirectional reactive current control if $k_{\text {sogi_dc }}$ can be reduced or kept low. This conclusion coincides with the simulations given in the last plot of Fig. 6 (b). As can be seen, for the case with a larger $k_{\text {sogi_dc }}$, i.e., from $\mathrm{C}_{1}$ to $\mathrm{C}_{2}$, the change of reactive current leads to an unstable system. By contrast, for the same reactive current change, the transition is stable when using a lower $k_{\text {sogi_dc }}$, as seen from the dc voltage waveform when $i_{q}^{r e f}$ is changed from $\mathrm{D}_{1}$ to $\mathrm{D}_{2}$.

Similarly, reducing $k_{\text {sogi_dc }}$ to gain margins on stability may deteriorate the dynamic performance of the peak dc voltage detection. If the compromises on the dynamic of dc voltage are undesirable, it is expected to develop ancillary controls for stability enhancement, or, formulating the problem of balancing the dynamic response of dc voltage and the system's stability as an optimization problem, by which a more appropriate set of control parameters could be found. Although these topics are interesting, they are outside the scope of this paper's analysis.

\section{IV.EXPERIMENTAL VERIFICATIONS}

The credibility of the presented stability analysis relies on the accuracy of the algorithmically generated models, which should be validated. Thus, in the following, both frequency-domain and time-domain experiments will be conducted to verify the effectiveness of the presented method. 


\section{A. Experimental setup}

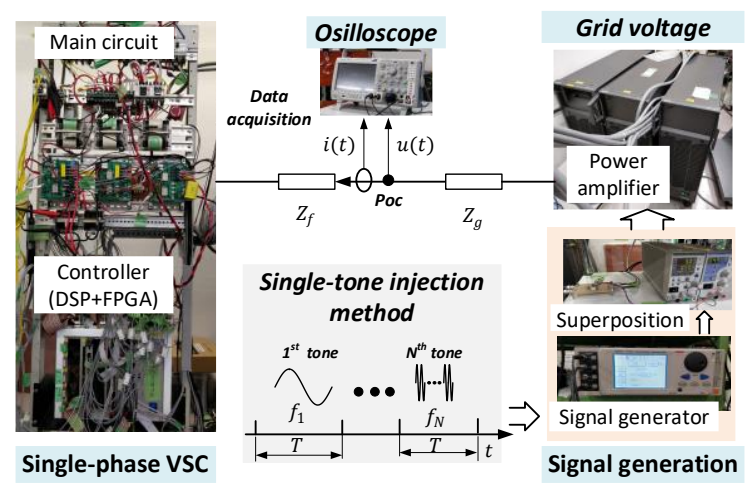

Fig. 7 Experimental setup for the frequency-scan and stability tests

The experimental setup is shown in Fig. 7 primarily consisting of a signal generator, a linear power amplifier, and a single-phase VSC. The VSC control is digitally implemented in the PE-expert4 platform, where the control cycle $T_{d}$ is 50 us and the converter switching frequency $f_{s w}$ is $10 \mathrm{kHz}$.

For verifying the small-signal models by frequency-scans, the single-tone injection-based method is applied. This means

for a specific time-interval $T$, only one specified frequency is injected into the system. Then, this process is repeated, where the frequency is swept from $5 \mathrm{~Hz}$ to $1 \mathrm{kHz}$. The time interval $T$ used in this experiment is $6 \mathrm{~s}$ to ensure the system reach the steady-state before the subsequent perturbation is imposed. The perturbation voltage perturbation together with the supplying voltage is generated by the power amplifier. Besides, voltage and current data are collected and recorded by the oscilloscope (the sampling rate is $50 \mathrm{kHz}$, and the window for discrete Fourier analysis is $5 \mathrm{~s}$ ). For conducting the time-domain stability tests, the signal generator used in frequency scan is disabled.

\section{B. Model verification via experimental frequency scan}

To verify the HSS model (3) used for stability assessments, its input-output formation will be used, which is in fact a harmonic transfer function (HTF) written as:

$$
\Delta \mathcal{Y}=\underbrace{\left[\mathcal{C}_{\mathrm{vsc}}^{(i)}\left(s \mathcal{I}-\mathcal{A}_{\mathrm{vsc}}{ }^{(i)}+\mathcal{N}_{\mathrm{blk}}\right)^{-1} \mathcal{B}_{\mathrm{vsc}}^{(i)}+\mathcal{D}_{\mathrm{vsc}}^{(i)}\right]}_{\mathcal{H}_{\mathrm{scc}}(s)} \cdot \Delta \mathcal{U}
$$

where $\Delta \mathcal{Y}, \Delta \mathcal{U}$ are the spectral vectors of $i_{a}, u_{a}$ in Fig. 1 (a), thus $\mathcal{H}_{\mathrm{vsc}}(s)$ denotes the VSC admittance. All the required
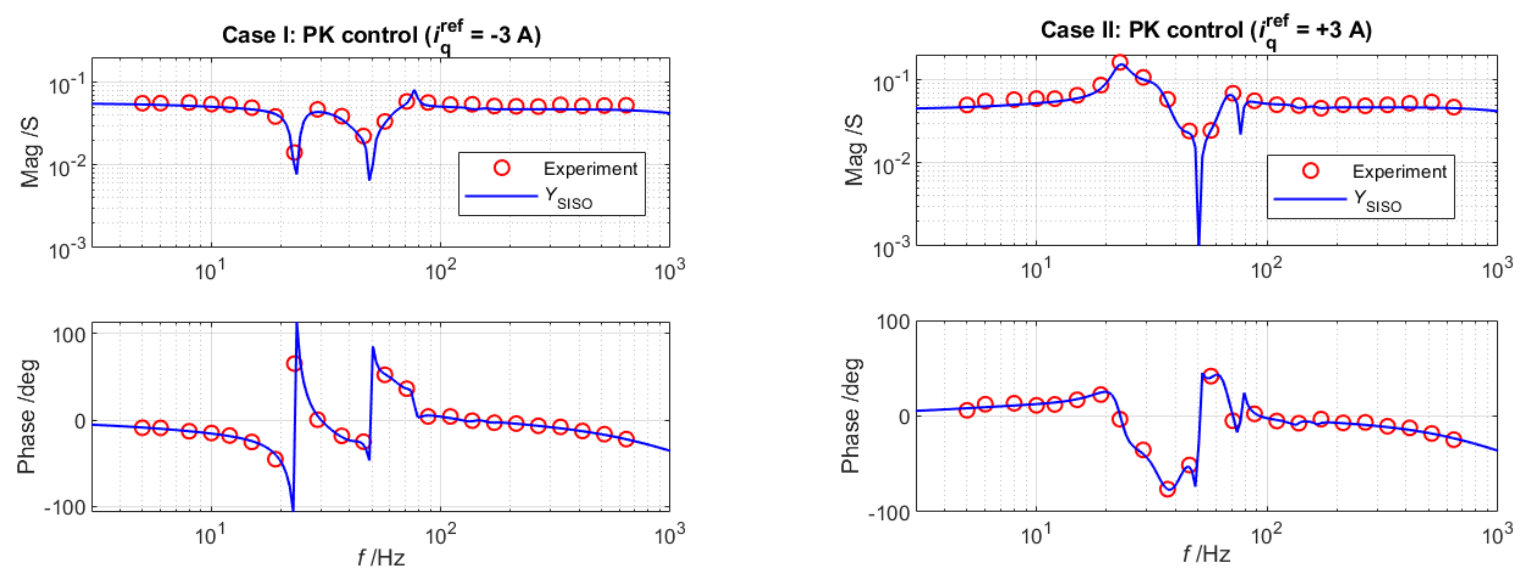

(a) Verification for the PK control under two types of reactive currents
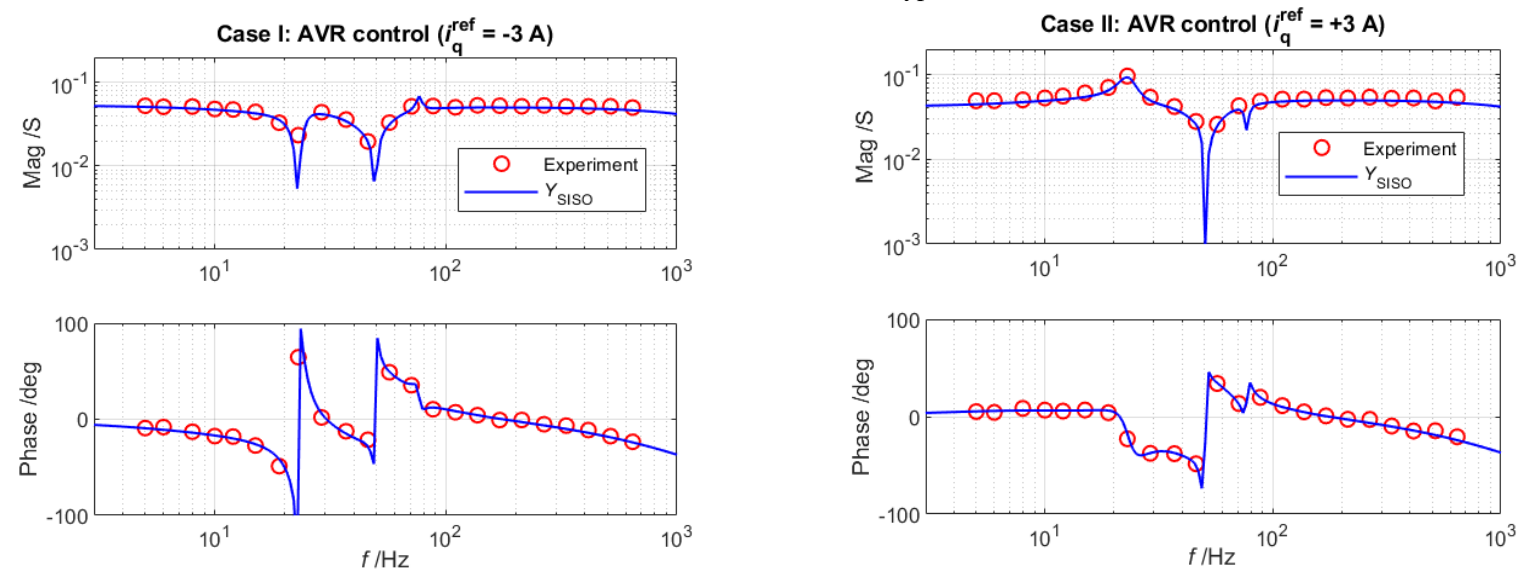

(b) Verification for the Avr control under two types of reactive currents

Fig. 8 Model verification via experimental frequency-scan. Main parameters are given in Table III.

Table III Experimental control and circuit parameters

\begin{tabular}{l|l|l|l|l|l|l|l|l}
\hline \hline Name & $k_{p d c} ; k_{i d c}$ & $k_{p c} ; k_{i c}$ & $k_{p p l l} ; k_{i p l l}$ & $k_{\text {sogi_dc }}$ & $k_{\text {sogi }}$ & $T_{d}$ & $f_{s w}$ & Circuit parameters \\
\hline Values & $5 \times 10^{-5} ; 2.5 \times 10^{-4}$ & $20 ; 2 \omega_{1}$ & $0.1 ; 100$ & 5 & 5 & $50 \mu s$ & $10 \mathrm{kHz}$ & Table II \\
\hline Note: $k_{p d c}$ and $k_{i d c}$ are much smaller than those shown in Table II because of the scaling effects in measurements (e.g., the sampling units), and the scaling \\
factor is around 1414. Also, different from the bandwidth convention used in simulations, $k_{p c}, k_{i c}$ in experiment are regarded as two independent parameters. \\
\hline
\end{tabular}


matrices can be obtained from the iteration subroutine in Fig. 3. As $\mathcal{H}_{\text {vsc }}(s)$ is a multi-input and multi-output (MIMO) system, its direct verification via the frequency scan is cumbersome, where a large number of perturbations is required, e.g., for a $2 N+1$ dimensional HTF, $2 N+1$ times of injections are needed for measuring the frequency response of the HTF at each frequency point [19]. To allow a simpler verification, $\mathcal{H}_{\mathrm{vsc}}(s)$ can be first converted into a single-input and single-output (SISO) equivalent model $Y_{\text {SISO }}(s)$ according to [28]. Then, $Y_{\text {SISO }}(s)$ can be verified instead of $\mathcal{H}_{\text {vsc }}(s)$ so that the typical frequency scan routine as illustrated above can be applied.

Based on this method, the frequency responses of $Y_{\text {SISO }}(s)$ under Avr and PK controls will be compared with experiments, where the following two cases are tested: 1) Case I: Inject reactive power by controlling a negative reactive current, i.e., $i_{q}^{\text {ref }}<0$; 2) Case II: Absorb reactive power by controlling a positive reactive current, i.e., $i_{q}^{r e f}>0$.

According to the comparative results given in Fig. 8 (a), it can be seen that the frequency responses of PK control under both of the cases are consistent with experiments, and the same conclusion applies to Avr control, as indicated by the results in Fig. 8 (b). Overall, this experimental frequency scan indicates that the generated models using the presented method are valid, which in turn justifies the validity of the harmonic-order $N$ used in the iteration model (as is introduced in Section II.C).
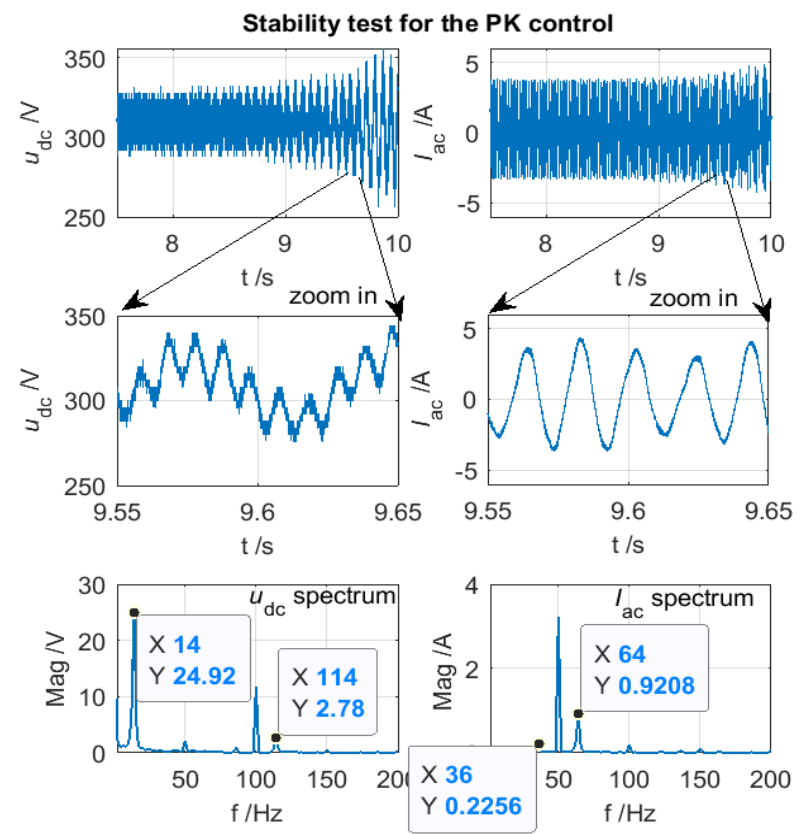

\section{Experimental stability tests and analysis}

To consolidate the frequency-domain analysis, time-domain experimental stability tests will be presented. As a starting point, the theoretically predicted weakest modes using the presented method are shown in Fig. 9 for the PK and Avr control, with the parameters and main results given in Table IV.
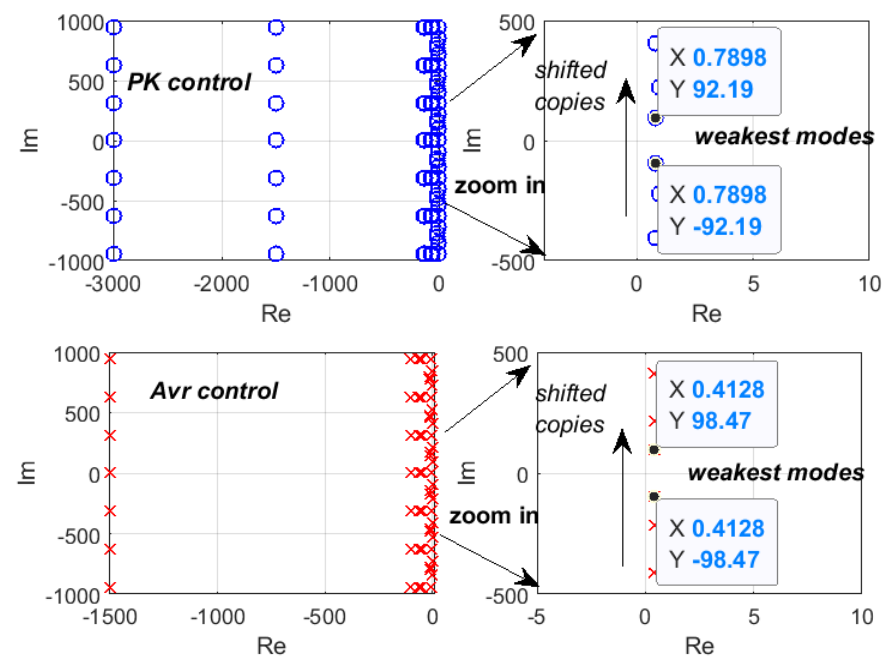

Fig. 9 Eigenvalue plots and weakest modes under the conditions of Table IV.

According to the obtained weakest modes, first, it can be predicted that both control strategies will result in an unstable system under the given conditions (because $\operatorname{Re}\left[\lambda_{\mathrm{wk}}\right]>0$ ). Moreover, the oscillation frequency under the PK control can
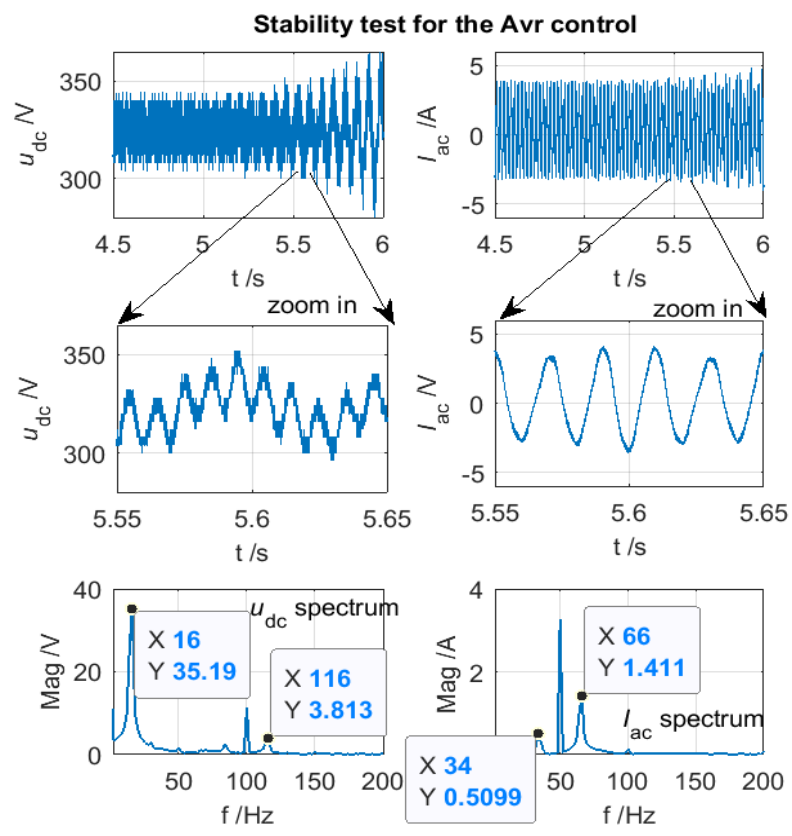

Fig. 10 Experimental stability tests and analysis. For the PK control, around $8 \mathrm{~s}, k_{p c}$ is changed from 20 to 1.5 (the condition in Table IV); the sepctra are obtained using the data between $9 \mathrm{~s}$ and $9.5 \mathrm{~s}$; the dc component of dc votlage is not shown for better presentation. For the Avr control, around 4.5s, $k_{p c}$ is changed from 20 to 1 (the condition in Table IV); the sepctra are obtained using the data between $5 \mathrm{~s}$ and $5.5 \mathrm{~s}$; for a similar reason, the dc component is not shown.

Table IV Conditions and theoretical stability results

\begin{tabular}{l|l|l|l|l|l|l|l}
\hline \hline \multicolumn{2}{c}{ PK control } & \multicolumn{3}{c}{ Avr control } \\
\hline$k_{p c}$ & $i_{q}^{\text {ref }}$ & $\lambda_{w k_{-} P K}$ & Stability & $k_{p c}$ & $i_{q}^{\text {ref }}$ & $\lambda_{w k_{A} A v r}$ & Stability \\
\hline 1.5 & $-3 \mathrm{~A}$ & $0.79 \pm \mathrm{j}\left(92.2 \pm k \omega_{1}\right)$ & Unstable & 1 & $-3 \mathrm{~A}$ & $0.41 \pm \mathrm{j}\left(98.5 \pm k \omega_{1}\right)$ & Unstable \\
\hline
\end{tabular}


be estimated by: $f_{\text {os__PK }}=(14.67 \pm 50 k) H z, k=0,1,2, \ldots$ (i.e., from $\operatorname{Im}\left[\lambda_{\mathrm{wk}}\right]$ ), while for the Avr control, it is estimated as: $f_{\text {os__PK }}=(15.67 \pm 50 \mathrm{k}) \mathrm{Hz}$.

To verify these theoretical stability results, time-domain stability tests are conducted using the conditions as Fig. 9. According to the experimental results in Fig. 10, it can be clearly seen that when the control parameters of the Avr and PK controls are set to those given in Table IVTable III, small-signal instability in the form of oscillations occurs for both systems.

Further, by inspecting the spectra of dc voltage and ac current of the PK control, it can be obtained that the oscillation modes are frequency shifted copies of $14 \mathrm{~Hz}$, which is very close to the theoretical result, i.e., frequency shifted copies of $14.67 \mathrm{~Hz}$. On the other hand, the oscillation modes of the Avr control from experiments are shown to consist of frequency-shifted copies of $16 \mathrm{~Hz}$, which is also very close to its predictions, i.e., frequency-shifted copies of $15.67 \mathrm{~Hz}$.

Therefore, the experimental stability tests further confirm the validity and accuracy of the presented method, thus ensuring the credibility of the conclusions obtained in this paper.

\section{CONCLUSIONS}

This paper presents a comprehensive stability analysis and comparison of the single-phase STATCOM using the PK and Avr control. Apart from the known merits of PK control in enabling reduced dc-side capacitance, this paper achieved an indepth understanding of the PK control in view of its parametric stability performance. The main conclusions and findings are:

1) Under non-ideal grids, the $P K$ control is prone to be unstable when absorbing reactive power, as a consequence of high sensitivity to some of its control parameters, e.g., $k_{\text {sogi_dc }}$ and $k_{p d c}$. By contrast, the Avr control is shown to be less susceptible to this issue and overall exhibits a better stability performance than the PK control.

2) Control parameter design of PK control using ideal grid assumption should be of great caution, particularly for those parameters identified to be sensitive to grid condition changes, e.g., $k_{\text {sogi_dc }}$ and $k_{p d c}$. Otherwise, the designed system cannot be ensured to be stable when connected to non-ideal grids.

3) Alleviating the potential stability risks of the PK control can be achieved by properly designing the control parameters, which can be assisted by the presented stability plot.

Besides, according to the various analyses presented in this paper, it also demonstrates that the developed tool has a great potential of being promoted to other PSS-based converter systems for fast and efficient parametric stability tests.

\section{APPENDIX}

\section{A. State-space model under Avr control}

State-space modeling of the grid-VSC system can be fulfilled by first listing all the state equations of controllers and circuit elements; then connecting them via control diagrams and basic circuit laws. Since this process is straightforward (which can be readily obtained from Fig. 1 (a) and (b)), only the final results along with necessary explanations will be given in the next.

\section{1) SOGI-QSG for synchronization}

$$
\left[\begin{array}{l}
\dot{x}_{\text {sogia }} \\
\dot{x}_{\text {sogib }}
\end{array}\right]=\left[\begin{array}{l}
\omega_{1} \cdot k_{\text {sogi }}\left(u_{a}-\hat{u}_{a}\right)-\omega_{1} \cdot \hat{u}_{\mathrm{b}} \\
\hat{u}_{a}
\end{array}\right]=f_{\text {sogi_syn }}
$$

where the in-phase and quadrature outputs of the SOGI-QSG are $\hat{u}_{a}=x_{\text {sogia }}$ and $\hat{u}_{\mathrm{b}}=\omega_{1} x_{\text {sogib }}$.

2) $S R F-P L L$

$$
\left[\begin{array}{l}
\dot{\delta}_{\mathrm{pll}} \\
\dot{x}_{\mathrm{pll}}
\end{array}\right]=\left[\begin{array}{l}
k_{\mathrm{ppll}} \cdot u_{q}+x_{\mathrm{pll}} \\
k_{\mathrm{ipll}} \cdot u_{\mathrm{q}}
\end{array}\right]=\boldsymbol{f}_{\mathrm{PLL}}
$$

where $u_{\mathrm{q}}=-\sin \left(\delta_{\mathrm{pll}}+\omega_{\mathrm{l}} t\right) \cdot \hat{u}_{\mathrm{a}}+\cos \left(\delta_{\mathrm{pll}}+\omega_{1} t\right) \cdot \hat{u}_{\mathrm{b}}$. In which, the substitution $\delta_{\mathrm{pll}}=\theta_{\mathrm{pll}}-\omega_{\mathrm{l}} t$ is used.

3) dc voltage and controller

$$
\dot{x}_{\mathrm{dc}}=k_{\mathrm{idc}} \cdot\left(u_{\mathrm{dc}}^{2}-V_{\mathrm{dc} \_ \text {ref }}^{2}\right)=f_{\mathrm{dc} \_ \text {Avr }}
$$

where the output is $i_{\mathrm{d}}^{\mathrm{ref}}=k_{\mathrm{pdc}}\left(u_{\mathrm{dc}}^{2}-V_{\mathrm{dc} \_ \text {ref }}^{2}\right)+x_{\mathrm{dc}}$.

4) PR-based current control

$$
\left[\begin{array}{l}
\dot{x}_{\mathrm{pra}} \\
\dot{x}_{\mathrm{prb}}
\end{array}\right]=\left[\begin{array}{l}
\omega_{1} \cdot\left(i_{\mathrm{a}}^{\mathrm{ref}}-i_{\mathrm{a}}-\omega_{1} x_{\mathrm{prb}}\right) \\
x_{\mathrm{pra}}
\end{array}\right]=\boldsymbol{f}_{\mathrm{PR}}
$$

where $i_{\mathrm{a}}^{\text {ref }}=i_{\mathrm{d}}^{\text {ref }} \cos \left(\omega_{1} t+\delta_{\mathrm{pll}}\right)-i_{\mathrm{q}}^{\text {ref }} \sin \left(\omega_{1} t+\delta_{\mathrm{pll}}\right)$, and the current PR controller output is:

$$
m_{\mathrm{a}}^{\mathrm{ref}}=\frac{1}{u_{\mathrm{dc}}}\left[k_{\mathrm{pc}} \cdot\left(i_{\mathrm{a}}^{\mathrm{ref}}-i_{\mathrm{a}}\right)+\frac{2 k_{\mathrm{ic}}}{\omega_{1}} \cdot x_{\mathrm{pra}}\right]
$$

where $m_{a}^{r e f}$ is the modulation signal as shown in Fig. 1 (a).

5) Circuit model

The circuit model shown below includes the dc capacitor, ac/dc modulation, converter filter as well as the grid impedance.

$$
\left[\begin{array}{l}
\dot{u}_{\mathrm{dc}} \\
\dot{i}_{\mathrm{a}}
\end{array}\right]=\left[-\frac{m_{\mathrm{a}}^{\mathrm{ref}} i_{\mathrm{a}}}{C_{\mathrm{cap}}}, \frac{m_{\mathrm{a}}^{\mathrm{ref}} u_{\mathrm{dc}}-u_{\mathrm{g}}-\left(R_{\mathrm{f}}+R_{\mathrm{g}}\right) i_{\mathrm{a}}}{L_{\mathrm{f}}+L_{g}}\right]^{T}=f_{\text {cir }}
$$

In which, the PoC voltage used in (A.1) can be obtained as:

$$
u_{\mathrm{a}}=k_{\mathrm{f}} u_{\mathrm{g}}+\left(k_{\mathrm{f}} R_{\mathrm{g}}-k_{\mathrm{g}} R_{\mathrm{f}}\right) i_{\mathrm{a}}+k_{\mathrm{g}} m_{\mathrm{a}}^{\text {ref }} u_{\mathrm{dc}}
$$

where $k_{\mathrm{f}}=L_{\mathrm{f}} /\left(L_{\mathrm{f}}+L_{g}\right), k_{\mathrm{g}}=1-k_{\mathrm{f}}$.

Combing (A.1)-(A.7) will result in the final closed-loop model of the Avr control in a similar formation of (1):

$$
\begin{aligned}
& \dot{\boldsymbol{x}}=\boldsymbol{f}_{\text {cl_Avr }}(\boldsymbol{x}, \boldsymbol{u}, t) \\
& \boldsymbol{y}=\boldsymbol{g}_{\text {cl_Avr }}(\boldsymbol{x}, \boldsymbol{u}, t)
\end{aligned}
$$

where $\boldsymbol{g}_{\text {cl_Avr }}=\boldsymbol{C} \boldsymbol{x}, \boldsymbol{u}=\left[V_{\mathrm{dc} \_ \text {ref }}^{2}, i_{\mathrm{q}}^{\text {ref }}, u_{\mathrm{g}}\right]$, and $\boldsymbol{C}=\left[\boldsymbol{0}_{1 \times 4}, 1, \boldsymbol{0}_{1 \times 4}\right]$

$\boldsymbol{x}=\left[x_{\mathrm{dc}}, x_{\mathrm{pra}}, x_{\mathrm{prb}}, u_{\mathrm{dc}}, i_{\mathrm{a}}, x_{\mathrm{sogia}}, x_{\mathrm{sogib}}, \delta_{\mathrm{pll}}, x_{\mathrm{pll}}\right]^{T}$

$\boldsymbol{f}_{\mathrm{cl} \_\mathrm{Avr}}=\left[f_{\mathrm{dc} \_\mathrm{Avr}}, \boldsymbol{f}_{\mathrm{PR}}, f_{\mathrm{cir}}, \boldsymbol{f}_{\text {sogi_syn }}, \boldsymbol{f}_{\mathrm{PLL}}\right]^{T}$.

\section{B. State-space model under PK control}

The modeling of PK control is similar to that of Avr control. One major difference is the adoption of the SOGI-based filter (see Fig. 2 (a)) for peak-value dc voltage estimation (i.e., $\bar{u}_{\mathrm{dc} \_p k}^{2}$ ). The applied SOGI-QSG in the filter has the same structure as (A.1), which can be written as: 


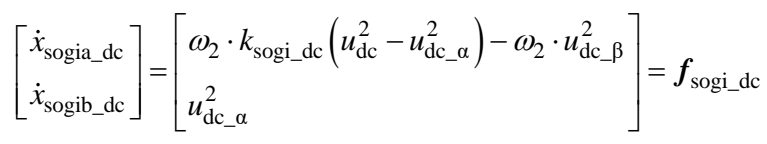

where $\omega_{2}=2 \omega_{1}, u_{\mathrm{dc} \_\alpha}^{2}=x_{\text {sogia_dc }}, u_{\mathrm{dc} \_\beta}^{2}=\omega_{2} x_{\text {sogib_dc }}$. The output of the filter (i.e., $\bar{u}_{\mathrm{dc} \_ \text {pk }}^{2}$ ) can be represented as:

$$
\bar{u}_{\mathrm{dc} \_\mathrm{pk}}^{2}=g_{\mathrm{pk}}\left(u_{\mathrm{dc}}^{2}, u_{\mathrm{dc} \_\alpha}^{2}, u_{\mathrm{dc} \_\beta}^{2}, k_{\mathrm{sogi} \_\mathrm{dc}}\right)
$$

where function $g_{\mathrm{pk}}(\cdot)$ denotes the result of algebraic operations performed on the inputs (according to Fig. 2 (a)). Given this output, (A.3) is modified as:

$$
\dot{x}_{\mathrm{dc}}=k_{\mathrm{idc}} \cdot\left(\bar{u}_{\mathrm{dc} \_\mathrm{pk}}^{2}-V_{\mathrm{dc} \_ \text {ref }}^{2}\right)=f_{\mathrm{dc} \_ \text {PK }}
$$

Combining (B.1)-(B.3), (A.2), and (A.4)-(A.7) results in the final closed-loop model of PK control similar to (1):

$$
\begin{aligned}
\dot{\boldsymbol{x}} & =\boldsymbol{f}_{\text {cl_PK }}(\boldsymbol{x}, \boldsymbol{u}, t) \\
\boldsymbol{y} & =\boldsymbol{g}_{\text {cl_PK }}(\boldsymbol{x}, \boldsymbol{u}, t)
\end{aligned}
$$

where $\boldsymbol{g}_{\text {cl_PK }}=\boldsymbol{C} \boldsymbol{x}, \boldsymbol{u}=\left[V_{\mathrm{dc} \_ \text {ref }}^{2}, i_{\mathrm{q}}^{\text {ref }}, u_{\mathrm{g}}\right]$, and $\boldsymbol{C}=\left[\boldsymbol{0}_{1 \times 6}, 1, \boldsymbol{0}_{1 \times 4}\right]$. $\boldsymbol{x}=\left[x_{\text {sogia_dc }}, x_{\text {sogib_dc }}, x_{\mathrm{dc}}, x_{\text {pra }}, x_{\text {prb }}, u_{\mathrm{dc}}, i_{\mathrm{a}}, x_{\text {sogia }}, x_{\text {sogib }}, \delta_{\text {pll }}, x_{\text {pll }}\right]^{T}$, $\boldsymbol{f}_{\text {cl_PK }}=\left[\boldsymbol{f}_{\text {sogi_dc }}, f_{\text {dc_PK }}, \boldsymbol{f}_{\mathrm{PR}}, f_{\text {cir }}, \boldsymbol{f}_{\text {sogi_syn }}, \boldsymbol{f}_{\mathrm{PLL}}\right]^{T}$.

In this paper, (A.8) and (B.4) correspond to the generic system (1), i.e., the input of "model preparation subroutine".

\section{Definition of current control and PLL bandwidths}

In this paper, the relations between the defined closed-loop bandwidths and their controller gains are: $k_{\mathrm{pc}}=2 \alpha_{\mathrm{c}} L_{\mathrm{f}}, k_{\mathrm{ic}}=2 \alpha_{\mathrm{c}}^{2} L_{\mathrm{f}}$, $k_{\mathrm{ppll}}=2 \alpha_{\mathrm{pll}} / U_{N}, k_{\mathrm{ipll}}=2 \alpha_{\mathrm{pll}}^{2} / U_{N}$.

\section{REFERENCES}

[1] H. Liu, X. Xie, J. He, J, T. Xu, Z. Yu, C. Wang, C. Zhang, "Subsynchronous Interaction Between Direct-Drive PMSG Based Wind Farms and Weak AC Networks," in IEEE Transactions on Power Systems, vol. 32, no. 6, pp. 4708-4720, Nov. 2017.

[2] C. Li, "Unstable Operation of Photovoltaic Inverter from Field Experiences," in IEEE Transactions on Power Delivery, Vol. 33, No. 2, April 2018, pp. 1013-1015

[3] M. Belkhayat, "Stability criteria for AC power systems with regulated loads," Ph.D. dissertation, Purdue University, USA, 1997.

[4] J. Sun, "Impedance-Based Stability Criterion for Grid-Connected Inverters," in IEEE Transactions on Power Electronics, Vol. 26, No. 11, November 2011, pp. 3075-3078

[5] X. Wang and F. Blaabjerg, "Harmonic Stability in Power Electronic Based Power Systems: Concept, Modeling, and Analysis," in IEEE Transactions on Smart Grid, Vol. 10, No. 3, May 2019, pp. 2858-2870

[6] D. Dong, B. Wen, D. Boroyevich, P. Mattavelli and Y. Xue, "Analysis of Phase-Locked Loop Low-Frequency Stability in Three-Phase GridConnected Power Converters Considering Impedance Interactions," in IEEE Transactions on Industrial Electronics, vol. 62, no. 1, pp. 310-321, Jan. 2015.

[7] B. Wen, D. Boroyevich, R. Burgos, P. Mattavelli and Z. Shen, "SmallSignal Stability Analysis of Three-Phase AC Systems in the Presence of Constant Power Loads Based on Measured d-q Frame Impedances," in IEEE Transactions on Power Electronics, Vol. 30, No. 10, October 2015, pp. 5952-5963,

[8] M. Cespedes and J. Sun, "Impedance Modeling and Analysis of GridConnected Voltage-Source Converters," in IEEE Transactions on Power Electronics, Vol. 29, No. 3, pp. March 2014, 1254-1261

[9] X. Wang, L. Harnefors and F. Blaabjerg, "Unified Impedance Model of Grid-Connected Voltage-Source Converters," in IEEE Transactions on Power Electronics, Vol. 33, No. 2, February 2018, pp. 1775-1787
[10] A. Rygg, M. Molinas, C. Zhang and X. Cai, "A Modified SequenceDomain Impedance Definition and Its Equivalence to the dq-Domain Impedance Definition for the Stability Analysis of AC Power Electronic Systems," in IEEE Journal of Emerging and Selected Topics in Power Electronics, vol. 4, no. 4, Dec. 2016, pp. 1383-1396

[11] L. Harnefors, M. Bongiorno and S. Lundberg, "Input-Admittance Calculation and Shaping for Controlled Voltage-Source Converters," in IEEE Transactions on Industrial Electronics, vol. 54, no. 6, December 2007, pp. 3323-3334

[12] L. Harnefors, "Modeling of Three-Phase Dynamic Systems Using Complex Transfer Functions and Transfer Matrices," in IEEE Trans. Ind. Electron, vol. 54, no. 4, pp. 2239-2248, 2007.

[13] Z. W Yao, P. G. Therond, and B. Davat, "Frequency Characteristic of AC Power System", IFAC $12^{\text {th }}$ Triennial World Congress, Sydney, Australia, 1993, pp. 737-740.

[14] N. M. Wereley, Analysis and control of linear periodically time varying systems, Diss. Massachusetts Institute of Technology, 1990.

[15] P. Vanassche, G. Gielen and W. M. Sansen, "Systematic modeling and analysis of telecom frontends and their building blocks," Springer Science \& Business Media, 2006.

[16] E. Mollerstedt and B. Bernhardsson, "Out of control because of harmonics-an analysis of the harmonic response of an inverter locomotive," in IEEE Control Systems Magazine, vol. 20, no. 4, pp. 7081, Aug. 2000.

[17] Ö. C. Sakinci and J. Beerten, "Generalized Dynamic Phasor Modeling of the MMC for Small-Signal Stability Analysis," in IEEE Transactions on Power Delivery, vol. 34, no. 3, pp. 991-1000, June 2019.

[18] C. Guo, J. Yang and C. Zhao, "Investigation of Small-Signal Dynamics of Modular Multilevel Converter Under Unbalanced Grid Conditions," in IEEE Transactions on Industrial Electronics, vol. 66, no. 3, pp. 22692279, March 2019.

[19] V. Salis, A. Costabeber, S. M. Cox and P. Zanchetta, "Stability Assessment of Power-Converter-Based AC systems by LTP Theory: Eigenvalue Analysis and Harmonic Impedance Estimation," in IEEE Journal of Emerging and Selected Topics in Power Electronics, vol. 5, no. 4, December 2017, pp. 1513-1525

[20] Y. Liao, Z. Liu, H. Zhang and B. Wen, "Low-Frequency Stability Analysis of Single-Phase System With dq-Frame Impedance ApproachPart I: Impedance Modeling and Verification," in IEEE Transactions on Industry Applications, Vol. 54, No. 5, October 2018, pp. 4999-5011.

[21] H. Wang, W. Mingli and J. Sun, "Analysis of Low-Frequency Oscillation in Electric Railways Based on Small-Signal Modeling of Vehicle-Grid System in dq Frame," in IEEE Transactions on Power Electronics, vol. 30, no. 9, pp. 5318-5330, Sept. 2015.

[22] H. Zhang, Z. Liu and S. Wu, "Sequence Impedance Modeling and Stability Analysis of Single-Phase Converters," $201821^{\text {st }}$ International Conference on Electrical Machines and Systems, ICEMS 2018, Jeju, Korea, pp. 2234-2239.

[23] S. Shah and L. Parsa, "On impedance modeling of single-phase voltage source converters," 2016 IEEE Energy Conversion Congress and Exposition, ECCE 2016, Milwaukee, WI, 2016, pp. 1-8.

[24] J. Carter, C. J. Goodman and H. Zelaya, "Analysis of the single-phase four-quadrant PWM converter resulting in steady-state and small-signal dynamic models", IEE Proc. Elect. Power Appl., vol. 144, no. 4, pp. 241247, Jul. 1997.

[25] T. Isobe, D. Shiojima, K. Kato, Y. R. R. Hernandez and R. Shimada, "Full-Bridge Reactive Power Compensator With Minimized-Equipped Capacitor and Its Application to Static Var Compensator," in IEEE Transactions on Power Electronics, vol. 31, no. 1, pp. 224-234, Jan. 2016.

[26] T. Isobe, L. Zhang, H. Tadano, J. A. Suul and M. Molinas, "Control of DC-capacitor peak voltage in reduced capacitance single-phase STATCOM," IEEE 17th Workshop on Control and Modeling for Power Electronics, COMPEL 2016, Trondheim, pp. 1-8.

[27] Y. Sun, Y. Liu, M. Su, W. Xiong, J. Yang, "Review of Active Power Decoupling Topologies in Single-Phase Systems," in IEEE Transactions on Power Electronics, vol. 31, no. 7, July 2016, pp. 4778-4794.

[28] C. Zhang, M. Molinas, S. Føyen, J. A. Suul and T. Isobe, "An Integrated Method for Generating VSCs' Periodical Steady-State Conditions and HSS-Based Impedance Model," in IEEE Transactions on Power Delivery, vol. 35 , no. 5, pp. 2544-2547, Oct. 2020. 


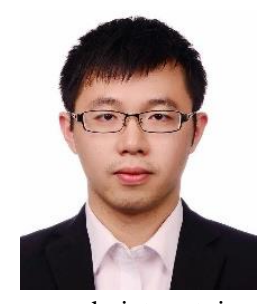

Chen Zhang received the B.Eng. degree from the China University of Mining and Technology, China, and the Ph.D. from Shanghai Jiao Tong University, China, in 2011 and 2018 respectively. He was a Postdoctoral Research Fellow at the Department of Engineering Cybernetics of NTNU, from March 2018 to October 2020. Currently, he is a postdoc with the Department of Electrical Engineering, Technical University of Denmark, Lyngby, Denmark. His research interest is modeling and stability analysis of VSC-based energy conversion systems, where the aim is to reveal the fundamental dynamics and stability mechanisms of renewable energies with VSCs as the grid interface.

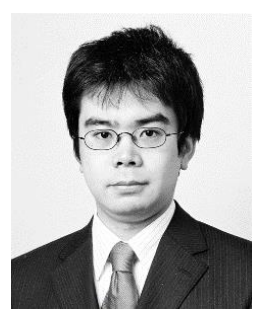

Takanori Isobe $\left(\mathrm{M}^{\prime} 07\right)$ was born in Hamamatsu, Japan, in 1978. He received the B.Eng. degree in physical electronics, the M.Eng. degree in nuclear engineering, and the D.Eng. degree in energy sciences from the Tokyo Institute of Technology, Tokyo, Japan, in 2003, 2005, and 2008, respectively. From 2008 to 2010 and from 2012 to 2013, he was a Researcher with the Tokyo Institute of Technology, where from 2010 to 2012, he was an Assistant Professor. From 2013 to 2014, he was with MERSTech, Tokyo. In 2013, he joined the University of Tsukuba, Tsukuba, Ibaraki, Japan, where he is currently an Associate Professor at the Faculty of Pure and Applied Sciences. His research interests include static reactive power compensators and softswitching power converters. Dr. Isobe is a Member of the Institute of Electrical Engineers of Japan and the Japan Institute of Power Electronics.

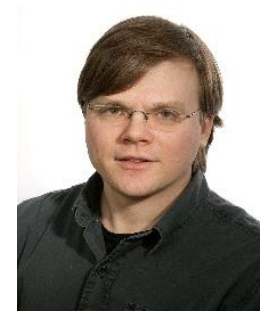

Jon Are Suul (M'11) received the M.Sc. degree in energy and environmental engineering and the Ph.D. degree in electric power engineering from the Norwegian University of Science and Technology (NTNU), Trondheim, Norway, in 2006 and 2012, respectively. From 2006 to 2007, he was with SINTEF Energy Research, Trondheim, where he was working with simulation of power electronic converters and marine propulsion systems until starting his Ph.D. studies. He returned to SINTEF Energy Research as a Research Scientist in 2012, first in a part-time position while working as a parttime Postdoctoral Researcher with the Department of Electric Power Engineering of NTNU until 2016. Since August 2017, he has been an Adjunct Associate Professor with the Department of Engineering Cybernetics, NTNU. His research interests are mainly related to modeling, analysis, and control of power electronic converters in power systems, renewable energy applications, and electrification of transport. He is Associate Editor in the IEEE Journal of Emerging and Selected Topics in Power Electronics and in the IEEE Journal of Emerging and Selected Topics in Industrial Electronics

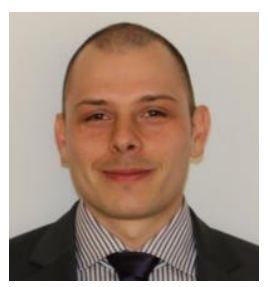

Tomislav Dragičević (S'09-M'13-SM'17) received the M.Sc. and the industrial Ph.D. degrees in Electrical Engineering from the Faculty of Electrical Engineering, University of Zagreb, Croatia, in 2009 and 2013, respectively. From 2013 until 2016 he has been a Postdoctoral researcher at Aalborg University, Denmark. From 2016 until 2020 he was an Associate Professor at Aalborg University, Denmark. Currently, he is a Professor at the Technical University of Denmark. He made a guest professor stay at Nottingham University, UK during spring/summer of 2018. His research interest is application of advanced control, optimization and artificial intelligence inspired techniques to provide innovative and effective solutions to emerging challenges in design, control and diagnostics of power electronics intensive electrical distributions systems and microgrids. He has authored and co-authored more than 300 technical publications (more than 150 of them are published in international journals, mostly in IEEE), 10 book chapters and a book in the field. $\mathrm{He}$ serves as an Associate Editor in the IEEE TRANSACTIONS ON INDUSTRIAL ELECTRONICS, in IEEE TRANSACTIONS ON POWER ELECTRONICS, in IEEE Emerging and Selected Topics in Power Electronics and in IEEE Industrial Electronics Magazine. Dr. Dragičević is a recipient of the Končar prize for the best industrial $\mathrm{PhD}$ thesis in Croatia, a Robert Mayer Energy Conservation award, and he is a winner of an Alexander von Humboldt fellowship for experienced researchers.

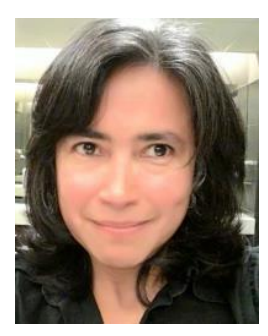

Marta Molinas (M'94) received the Diploma degree in electromechanical engineering from the National University of Asuncion, Asuncion, Paraguay, in 1992; the Master of Engineering degree from Ryukyu University, Japan, in 1997; and the Doctor of Engineering degree from the Tokyo Institute of Technology, Tokyo, Japan, in 2000. She was a Guest Researcher with the University of Padova, Padova, Italy, during 1998. From 2004 to 2007, she was a Postdoctoral Researcher with the Norwegian University of Science and Technology (NTNU) and from 2008-2014 she has been professor at the Department of Electric Power Engineering at the same university. She is currently Professor at the Department of Engineering Cybernetics, NTNU. Her research interests include stability of power electronics systems, harmonics, instantaneous frequency, and non-stationary signals from the human and the machine. She is Associate Editor for the IEEE Journal JESTPE, IEEE PELS Transactions and Editor of the IEEE Transactions on Energy Conversion. Dr. Molinas has been an AdCom Member of the IEEE Power Electronics Society from 2009 to 2011. 